\title{
Birthing Experiences of Spanish Speakers During the COVID-19 Pandemic in NYC
}

\author{
Stephanie Granada ${ }^{1,2}\left(\right.$ D $\cdot$ Cibel Quinteros Baumgart ${ }^{1} \cdot$ Devon Rupley $^{1}$
}

Accepted: 4 January 2022 / Published online: 8 February 2022

(c) The Author(s), under exclusive licence to Springer Science+Business Media, LLC, part of Springer Nature 2022

\begin{abstract}
Patient-provider language discordance is known to negatively affect patient experiences. This study describes the birth experience during COVID-19 among monolingual Spanish and bilingual Spanish/English speakers. Qualitative videoconference interviews with 15 monolingual Spanish and 15 bilingual Spanish/English patients that gave birth during the COVID-19 pandemic at a NYC tertiary-care hospital. 93\% of participants had a positive birthing experience. Common themes were quality of care, birth outcome, and supportive staff. $80 \%$ of patients lacked a support person postpartum. $27 \%$ of Spanish-only speakers felt that an interpreter should have been provided but was not, and $47 \%$ felt the experience would have been different if they spoke English. The patient birth experience is tied to birth outcomes and quality of care and remained positive during the beginning of the COVID-19 pandemic. Restrictions on support people during the intra- or postpartum impacted the birthing experience more than provider language discordance.
\end{abstract}

Keywords COVID-19 $\cdot$ Birth experience $\cdot$ Spanish $\cdot$ Language discordance

\section{Background}

Differences in preferred language between providers and patients impact healthcare delivery and patient perceptions of clinical care. For example, women with a non-English preferred language report negative birthing experience, even in settings where they speak limited English or when interpreter services are available [1]. Regarding Spanish-speakers, a 2018 study of English- and Spanish-speaking women at risk of preterm birth noted perceptions of disrespect, unmet information needs, and inconsistent social support from their healthcare providers [2]. However, negative birthing experiences are not unique to language-discordant populations. The 2019 Giving Voices to Mothers study found that $16 \%$ of women who give birth in the United States report mistreatment with rates higher among women of color: $32.8 \%$ for Indigenous women, 25\% for Hispanic women and $22.5 \%$ for Black women [3]. Additionally, a study in South

Stephanie Granada

granadas@chop.edu

1 Vagelos College of Physicians and Surgeons, Columbia University, New York, NY, US

2 Children's Hospital of Philadelphia, 3401 Civic Center Blvd, Philadelphia, PA, US
Florida found that Hispanic women are more likely to feel disrespected and experience communication problems than non-Hispanic women during prenatal care appointments [4].

Despite the groundwork created by these studies, there is a gap in the literature regarding the effects of language on the experience of pregnancy and giving birth. When the coronavirus pandemic first hit the United States in March 2020, New York City (NYC) was an epicenter for the country and the world [5]. In response, regulations around hospital visitation, in-person prenatal visits, and the presence of birthing partners were increased [6]. Data from the 2003 SARS outbreak show that pregnant people had higher anxiety state scores compared to those pregnant prior to the outbreak [7]. Data has also shown the impact visitation regulations have on patients: one study of 885 participants who gave birth between March and July 2020 in the United States found that while almost all patients had a birth partner, $61 \%$ of the sample reported that they had inadequate birth support due to the absence of doulas, friends, or family members, and higher levels of anxiety and depression [8].

Understanding how language discordance contributes to perceptions of negative birth experience is critical to improving maternal and pediatric healthcare as negative birthing experiences have repercussions extending beyond hospital discharge including impacts on mental health and 
mother-baby bonding [9]. This study sought to describe the experiences of people giving birth during the beginning of COVID-19 pandemic in an early epicenter of the pandemic, NYC, and to explore the differences of birthing experiences among Spanish-speaking and English-speaking patients.

\section{Methods}

\section{Participants}

This was a cross-sectional retrospective study using qualitative interviews with patients who gave birth during the peak of the COVID-19 pandemic in a tertiary care hospital in a predominantly Hispanic/Latinx NYC neighborhood. Participants were separated into two groups of 15 each based on monolingual Spanish or bilingual English/Spanish preferred language. This study was approved by Columbia's Institutional Review Board.

Participant inclusion criteria included: (1) age $>18$ years old, (2) received prenatal care and delivered at one tertiary care facility in NYC in April or May 2020, (3) had public insurance, (4) self-identified as a monolingual or bilingual Spanish speaker and (5) had an active e-mail address. Participants were recruited from a postpartum outreach database using a systematic random sample of patients with English or Spanish preferred language by week until recruitment quota was met. Patients were then asked if they spoke English only, Spanish only, or both to determine eligibility and group assignment. Participants were compensated with a $\$ 25$ Visa gift card.

\section{Data Collection and Measures}

Semi-structured 1:1 Zoom interviews were conducted in participants preferred language by one of two bilingual investigators between September and October 2020. Interview scripts included questions regarding: demographics and medical history, birthing experience and postpartum stay, interpreter services and language experiences, decisionmaking around delivery location, and impact of COVID19 on their experience. Patients are typically expected to deliver at the same care center where they received prenatal care, however, patients can deliver at any facility that accepts public insurance. Questions were adapted from the 2019 Giving Voices to Mothers and the 2005 Hispanic Women's Perceptions of Patient Centeredness During Prenatal Care studies $^{3,4}$. Outcomes included quality of birthing experience, interpreter-service usage and experience, and impact of language discordance and COVID-19 on the patient experience.

\section{Data Analysis}

Interview audio recordings were transcribed and translated by two bilingual investigators. Transcripts of the interviews were independently coded by both investigators to generate a codebook which was revised in an iterative fashion using a constant comparative method and updated and applied to all data. Codes were organized into common themes for long-form questions including: details on contributors to a positive or negative birth experience, effects of not having a support person during the birth or postpartum period, impact of COVID-19, and, for Spanish-speaking patients, differences in experience from English speakers.

\section{Results}

\section{Population Overview}

A total of 30 participants, 15 monolingual Spanish and 15 bilingual Spanish and English, were included in the study. Participants were similar in age-, race-, and support systemrelated demographics (Table 1). All (100\%) monolingual Spanish-speaking participants were born outside of the United States and 26\% were citizens, compared to $26 \%$ of bilingual participants being born outside the United States and $100 \%$ being citizens. Monolingual participants were more likely to be multiparous with $80 \%$ of monolinguals having a prior birth compared to $60 \%$ of bilinguals.

Bilingual patients had higher rates of complications and staying an additional night in the hospital after delivery. Two monolingual and two bilingual participants (13.3\% of participants) lacked a support person during their birth either due to fear of COVID exposure or confusion around hospital policies. Eighty percent $(\mathrm{N}=24)$ of participants lacked a support person during a portion or entire duration of the postpartum period due to hospital COVID-19 restrictions.

\section{Birthing Experience}

Overall, 93\% $(\mathrm{N}=28)$ of participants described their birth experience as positive with similar distributions between groups. The most common themes, for both groups, were the outcome of their delivery, quality of service, and supportive staff (Fig. 1). The most highly cited theme was the birth of a healthy baby, with monolingual Spanish-speakers being twice as likely (66\%) to associate it with a positive birthing experience compared to bilinguals (33\%).

"Everything worked out well, thank God. Other than that I had COVID, my daughter was in perfect condition, there was a lot of precaution, and I had excellent 
Table 1 Patient Demographics

\begin{tabular}{lll}
\hline Characteristics of study sample & & \\
\hline Sample characteristic & $\begin{array}{l}\text { Bilingual speakers } \\
\text { N=15 }\end{array}$ & $\begin{array}{l}\text { Monolingual } \\
\text { Spanish speaker } \\
\text { N=15 }\end{array}$ \\
& & 30 \\
\hline Average age & 30 & \\
Race & & 3 \\
White & 1 & 0 \\
Black & 3 & 0 \\
Native American & 1 & 13 \\
Othre & 10 & \\
Birth place & & 0 \\
USA & 11 & 15 \\
Latin American & 4 & 4 \\
Immigration status & & 9 \\
Citizen & 15 & 2 \\
Resident & 0 & 8 \\
Other/did not disclose & 0 & 2 \\
Average years in the US (if not born in US & 21 & 3 \\
Pregnancy history & & 2.3 \\
Primaparous & 6 & 2 \\
Multiparous & 9 & \\
Average people to household rooms ratio & 2.5 & \\
Number of participants who experienced complications & 4 & \\
Average number of hospital nights following delivery & 3 & \\
\hline
\end{tabular}

\section{Frequency of Common Themes Associated with Participants' Birthing Experiences}

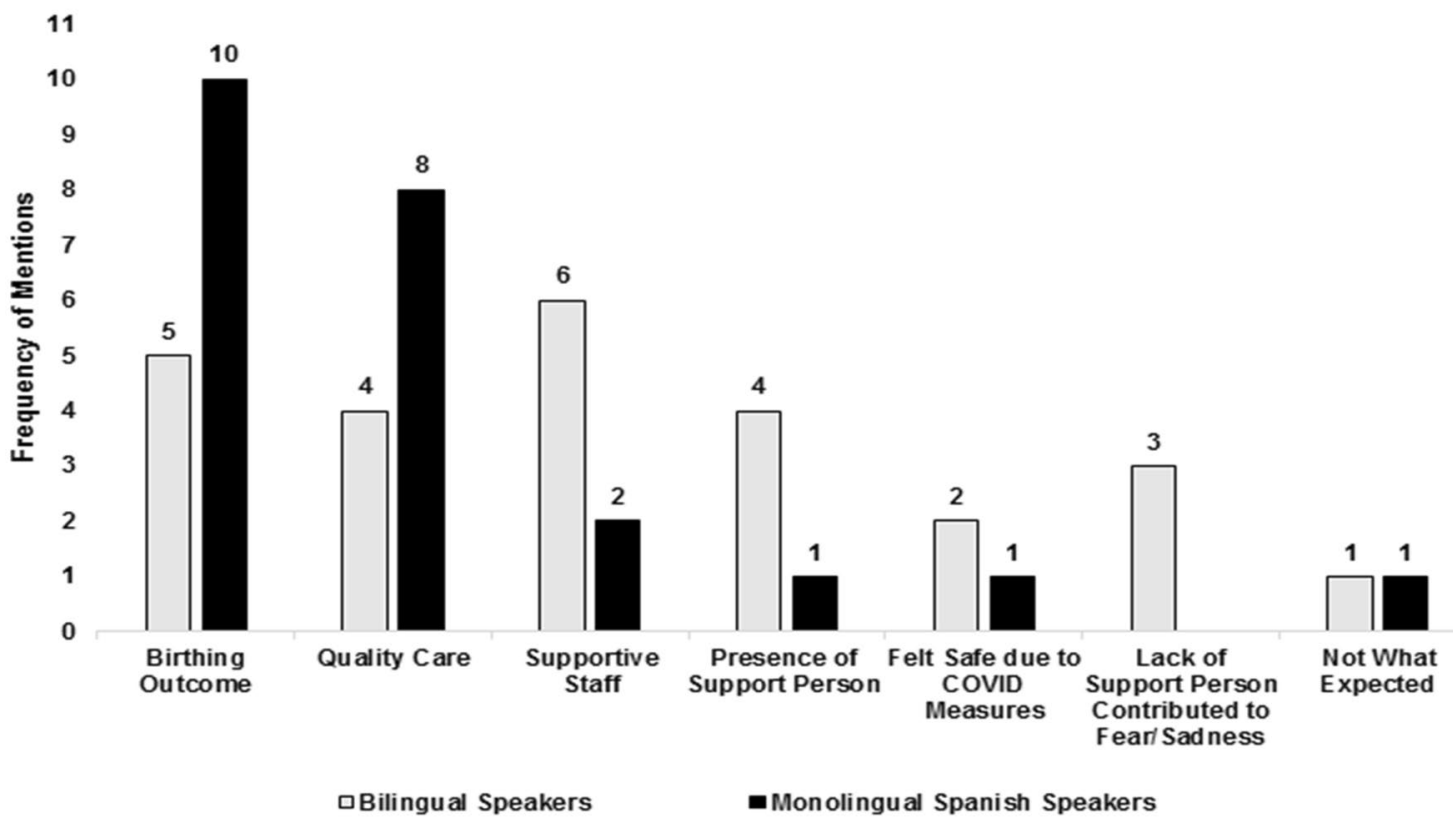

Fig. 1 Frequency of common themes associated with participants birthing experience. The most common factors that contributed to bilingual participants' experiences were, in descending order, being surrounded by a supportive staff, the outcome of their delivery, qual- ity care and the presence of a support person (partner, family member, or friend). Monolingual Spanish speakers cited birth outcome, the quality of care received, and the presence of a supportive staff as the most common contributors, in descending order 
care on the part of the doctors. Even though I couldn't have visitors or anyone, I never felt alone." - Monolingual Spanish Participant

The two participants who characterized their experience negatively did so because they were fearful of going to the hospital, were stressed about their and their baby's health in the pandemic and felt the birthing process was not what they expected.

"I would say bad delivery because I was going through a lot of stress at the moment... I felt like super anxious about just going to the hospital and being around people with the COVID situation... I was sort of scared at the moment for my health and the baby's health."

- Bilingual Participant

One bilingual speaker, accompanied by her husband, identified moments when she did not feel comfortable asking questions because she felt that providers expected her to already know the information, was not involved in her care without her doula to advocate for her, and felt providers were disrespectful, especially when treating her pain. One monolingual Spanish-speaker felt she did not understand the treatment options explained to her during her emergency C-section. All other participants, 90\% ( $=28)$ felt comfortable asking questions, understood their treatment options, felt involved in their care, and believed they were treated with respect by providers and staff.

\section{Language-related Experiences}

Eighty percent $(\mathrm{N}=12)$ of monolingual Spanish-speakers' preferred method of communication was Spanish-speaking doctors, $13 \%(\mathrm{~N}=2)$ preferred a phone interpreter, and $7 \%$ $(\mathrm{N}=1)$ preferred either option. 11 monolingual Spanishspeakers identified times when they had a fluent Spanishspeaking provider, but the duration of the interaction and level of fluency was not further probed.

Approximately $30 \%$ of monolingual Spanish-speakers felt there were points in their care when they needed an interpreter and lacked one. One bilingual speaker felt her care was impacted because an interpreter was not provided for her partner. Additionally, one bilingual participant and one monolingual participant felt there were times during their C-sections when they did not understand and/or did not receive important information about their care unrelated to language differences.

"Honestly I think that during my delivery there was not a single person that spoke Spanish. Not even for one minute."-Monolingual Spanish participant

"The last day when it was time to discharge me, yes there was one nurse who only spoke English and for which it was necessary to give me an interpreter...
She spoke to me in English... so I didn't know if [ the injection] was in the arm, trans muscular, or... I didn't

know” - Monolingual Spanish participant

Eighty percent $(\mathrm{N}=12)$ of monolingual Spanish-speakers had a positive interpreter experience. Three participants did not like using the interpreter because it was difficult to understand providers and uncomfortable to have an additional person involved, especially if this interpreter was male.

"I don't feel comfortable... Why have 3 people if you could have had a doctor that speaks Spanish for the Spanish speaking people that come in? ... a birth is complicated...in an emergency, how are you going to ask them for a minute? We can't wait a minute to call an interpreter." - Monolingual Spanish participant

Almost half of monolingual Spanish-speakers, 47\% $(\mathrm{N}=7)$ felt their experience would have been different had they spoken English and 60\% $(\mathrm{N}=9)$ of participants (four bilingual and five monolinguals) felt that doctors and nurses treat English- and Spanish-speaking patients differently. Sixty percent $(\mathrm{N}=9)$ of participants (six bilingual and three monolingual) felt that the healthcare system as a whole treats English- and Spanish-speaking patients differently.

"It would have been a lot easier [if I spoke English] because there are topics and terms in the medical sphere that you need to understand completely... If you don't speak English, or at least if you don't understand it in medical terms... You don't understand what is happening...you are going to keep saying okay, yes, or no, because you don't know." - Monolingual Spanish Participant

\section{Impact of COVID-19}

Most patients $(56.7 \%, \mathrm{~N}=17)$ felt that visitation restrictions that limited the presence of a support person, doula, or family member during the birth or postpartum period were the most significant way that COVID impacted their care (Fig. 2).

"It felt kind of lonely. Just me all by myself in there.... Imagine an 18-year-old with her first pregnancy, with her son having to stay in the NICU."-Bilingual participant

"I had dreamed that it would be different or that I would have my family and not be alone...it affected me $100 \%$... I couldn't begin a normal life with [my baby]."-Monolingual Spanish participant

"After having my c-section I would have liked if maybe I could have had my partner there with me, or you know, at least, my partner and my mom...but 


\section{Frequency of Common Themes Associated with COVID-19's Impact on Birthing} Experiences

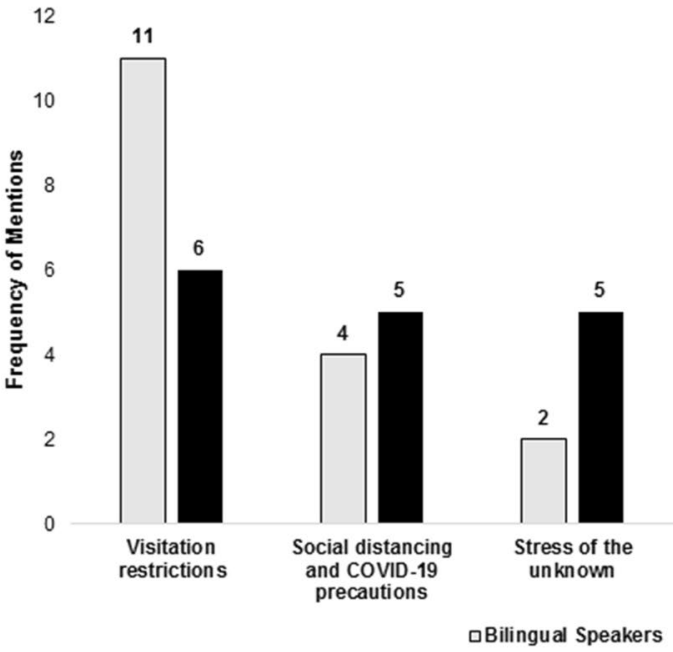

Fig.2 Frequency of common themes associated with COVID-19's impact on birthing Experiences. The most common way in which COVID-19 impacted both groups of participants was through hospital guidelines related to patient visitations, as well as self-imposed restrictions by participants to limit potential exposures in the hospital. The other most commonly cited themes for bilingual participants were having to be socially distant and follow other precautions dur-

that just made me feel really bad that I couldn't have them there."-Bilingual participant.

"My mom wasn't able to be there for my first baby, the first grandchild, so that was kind of um, kind of sad"-Bilingual participant

"I know that it would have been better if I didn't have COVID because I couldn't take off the mask, I couldn't even give my baby a kiss... it was so very painful."-Monolingual Spanish participant

Eight bilingual participants (53\%) and 11 monolingual participants (73\%) felt COVID-19 impacted their emotional well-being. All but one participant, a monolingual Spanish-speaker, felt they received quality care even in the pandemic. Nine participants noted dissatisfaction with telehealth during the antepartum period due to difficulty contacting providers, transitioning to video visits, and/or social distancing requirements at in-person visits. Five bilingual participants and two monolingual Spanishspeakers considered not giving birth in the hospital system where they received prenatal care due to concerns around COVID exposures in the hospital. No patients reported access to language services as a factor in deciding where to deliver, although this was not explicitly asked. Three bilingual participants and two monolingual participants felt that being in a single room impacted them emotionally due to feeling isolated and helped less by staff. ing the antepartum period, as well as the quality and frequency of antepartum visits and the inconveniences related to the COVID-19 test. In the monolingual Spanish-speaking group, the other commonly cited themes were also social distancing and COVID-19 precautions, as well as fears and anxieties related to the unprecedented nature of the pandemic

\section{Discussion}

We compared the birthing experiences of English- and Spanish-speaking patients during the beginning of the COVID-19 pandemic in NYC. Our study found that birthing experiences were positive overall, perceptions of a positive birthing experience were tied to the outcome of delivery, and that this association was stronger among monolingual Spanish-speakers. Our results differed from another United States study which found that patients who gave birth in COVID-19-exposed communities during March and April 2020 had increased risk of a traumatic childbirth experience compared to those who gave birth earlier in 2020 [10].

Lacking a support person due to visitation restrictions was the most profound way in which participants were affected, demonstrating the extent to which the healthcare system and patients rely on family members as part of the clinical care team and for emotional support. Pre-COVID studies have shown that at baseline, perceptions of partner support contribute to the birthing experience [11]. This is even more critical during a pandemic, as supported by a study from the United Kingdom demonstrating that partner presence was the most common theme discussed for intrapartum care for those pregnant or delivering during the pandemic [12]. This highlights how labor and delivery care is structurally set up to disadvantage patients without strong 
support networks in close physical proximity who can assist with physical and emotional needs during hospitalization.

Many participants were affected emotionally by COVID19 , citing feelings of anxiety, isolation, and fear, with rates $20 \%$ higher in our monolingual Spanish group. Poor maternal mental health is linked to worse maternal and neonatal outcomes globally, providing an area for potential intervention [13]. When thinking about the compounding impact of language discordance on perinatal stress, language-specific mental health interventions should be encouraged for vulnerable groups and providers should be aware of these disparities and have a low threshold for referral. While our study did not focus on the prenatal period, several participants described challenges during the antepartum period; this is an area for further study especially as the role of telehealth is expanded in antenatal care.

Many of our participants lacked an interpreter when they needed one, and others expressed difficulties with interpreter use. Lack of an interpreter or difficulties using an interpreter is an important aspect of the birth experience, as previous studies have found that Hispanic women are more likely to feel disrespected and experience communication problems than non-Hispanic women during prenatal care and are more likely to rely on a network of support persons to act as advocates and interpreters during childbirth [14]. While COVID-19 specific challenges seemed to be more impactful on patient experience than patient-provider language discordance in our study, the absence of in-person interpreters and patient-provided advocates (i.e., partners, family members, doulas) and its effect on the patient experience and potential adverse events cannot be overemphasized, as continuous support during the birthing process improves patient outcomes [15]. The frequency at which monolingual speakers felt their experience would have been different had they spoken English implies that language discordance remains a relevant factor in the care experience and in improving health outcomes in the setting of the maternal morbidity/ mortality crisis.

\section{Limitations}

This study had limited statistical power due to a small sample of 30 participants. Due to the demographics of the prenatal clinic we recruited from, we were only able to identify a limited number of monolingual English speakers and were unable to create a monolingual English comparison group due to lack of interest in those we approached. Our data may have also been affected by recall bias, as the interviews were held 4-5 months after the birth experience when the pandemic had evolved. We could not assess how the duration of fluent provider contact or how the level of provider fluency impacted the patient experience. Patient comfort with discussing negative perceptions of care could be impacted by basing the study out of the same institution where patients received care, despite assurance of confidentiality. Finally, this study only describes the experience of birthing at one institution.

\section{Conclusion}

Participants' perceived birth experience was associated with outcomes and quality of care and remained positive even during the COVID-19 pandemic in NYC. Restrictions on visitations and support persons during the birth or postpartum period caused emotional distress; this impacted the birthing experience more than provider language discordance. Ultimately, understanding the impact of the pandemic on the birth experience in all patients, but especially vulnerable populations, is critical to informing hospital regulations to maximize safety while supporting patients in future public health crises.

Acknowledgements This study was one of the first of its kind to characterize the birth experience in the beginning of the COVID-19 pandemic in the epicenter of New York City. Despite initial assumptions that language discordance would have a heightened impact during the pandemic, our data showed that COVID-19 specific challenges such as visitation and birthing partner restriction were more impactful than patient-provider language discordance and quality of birthing experiences were not significantly different between monolingual Spanish and bilingual English and Spanish-speakers overall. To improve the healthcare system for all patients and tackle health disparities, it is important that these relationships are further characterized in future studies.

Funding This research did not receive any specific grant from funding agencies in the public, commercial, or not-for-profit sectors.

\section{References}

1. Straus L, McEwen A, Hussein FM. Somali women's experience of childbirth in the UK: perspectives from somali health workers. Midwifery. 2009;25(2):181-6.

2. McLemore MR, et al. Health care experiences of pregnant, birthing and postnatal women of color at risk for preterm birth. Soc Sci Med. 2018;201:127-35.

3. Vedam S, et al. The Giving Voice to Mothers study: inequity and mistreatment during pregnancy and childbirth in the United States. Reprod health. 2019;16(1):77.

4. Darius TS, Parillo KM, Keefer M. Hispanic women's perceptions of patient-centeredness during prenatal care: a mixed-method study a. Birth. 2005;32(4):312-7.

5. Thompson NC, et al. COVID-19 Outbreak-New York City, February 29-June 1, 2020. Morb Mortal Wkly Rep. 2020;69(46):1725.

6. Caron C, Van Syckle K. Some pregnant women in New York City will have to deliver babies alone. New York Times. 2020;3:24.

7. Dominic TC, et al. Psychological responses of pregnant women to an infectious outbreak: a case-control study of the 2003 SARS outbreak in Hong Kong. J psychosom res. 2006;61(5):707-13. 
8. Mollard E, Wittmaack A. Experiences of Women Who Gave Birth in US Hospitals During the COVID-19 Pandemic. J Patient Exp. 2021;8:2374373520981492.

9. Reed R, Sharman R, Inglis C. Women's descriptions of childbirth trauma relating to care provider actions and interactions. BMC Pregnancy Childbirth. 2017;17(1):21.

10. Mayopoulos GA, et al. COVID-19 is associated with traumatic childbirth and subsequent mother-infant bonding problems. J Affect Disord. 2021;282:122-5.

11. Bryanton J, et al. Predictors of Women's Perceptions of the Childbirth Experience. J Obstet Gynecol Neonatal Nurs. 2008;37(1):24-34.

12. Karavadra B, et al. Women's perceptions of COVID-19 and their healthcare experiences: a qualitative thematic analysis of a national survey of pregnant women in the United Kingdom. BMC Pregnancy and Childbirth. 2020;20(1):1-8.
13. Atif $\mathrm{N}$, et al. Maternal mental health: the missing " $\mathrm{m}$ " in the global maternal and child health agenda. Semin perinatol. 2015;39(5):345-52.

14. Martin JA, et al. Births: final data for 2018. Natl vital stat rep. 2019;68:13.

15. Bohren MA, et al. Continuous support for women during childbirth. Cochrane database of systematic reviews. 2017. https://doi. org/10.1002/14651858.CD003766.pub6.

Publisher's Note Springer Nature remains neutral with regard to jurisdictional claims in published maps and institutional affiliations. 